\title{
Das steht in der neuen Hausarzt-Leitlinie
}

\section{Die neue S3-Leitlinie Multimorbidität der Deutschen Gesellschaft für Allge- meinmedizin stellt den multimorbiden Patienten als „großes Ganzes" in den Mittelpunkt - und gibt Ärzten eine Ge- sprächsanleitung an die Hand.}

— „Unsere Leitlinie soll vor einer kumulierten Anwendung monomorbider Leitlinien schützen, indem sie das, große Ganze in den Mittelpunkt rückt", heißt es in der Einführung. Und dazu gehört, wie es die Autoren um DEGAM-Vizepräsident Prof. Martin Scherer formuliert, der bewusste Verzicht auf diagnostische und therapeutische Prozeduren, die keinen relevanten Effekt auf die Gesamtsituation des Patienten haben, sondern, obwohl jeweils für sich genommen leitliniengetreu, zusammengenommen in Konflikt miteinander geraten.

Stattdessen wird dem Gespräch mit dem Patienten ausdrücklich die zentrale Rolle im Behandlungsprozess zugeschrieben. Dafür liefert die DEGAM nun ein Schema, genannt „Meta-Algorithmus“, das den „übergeordneten hausärztlichen Denkprozess“ abbilden soll. Die darin aufgeschlüsselten Entscheidungswege sollen als genereller Wegweiser dienen.

So sollen Ärzte beim Gespräch mit multimorbiden $\mathrm{Pa}$ tienten nun leitliniengerecht vorgehen:

- Aktueller Beratungsanlass: Das aktuelle Symptom darauf überprüfen, ob es im Zusam- menhang mit einer bekannten Ursache/Diagnose steht.

- Wenn ja: Einordnung und Überleitung in ein übergreifendes Krankheitsmanagement (unter anderem Medikamentenreview, schnittstellenbedingte Problemlagen) oder in ein problemspezifisches Management (mit Leitlinien für Einzeldiagnosen als Grundlage).

- Wenn nein: Identifikation der Ursache, Ausschluss von abwendbar gefährlichen Verläufen wie krankheitsbedingten Komplikationen, unerwünschten Arzneimittelwirkungen und Autonomieverlust.

„Eine wesentliche Voraussetzung für die Bewältigung komplexer Problemlagen bei Multimorbidität ist hinreichend Zeit für die Kommunikation zwischen Arzt und Patient", heißt es in der Präambel der Leitlinie. Politik, Ärzte und Gesellschaft müssten entsprechende Voraussetzungen schaffen.

Anne Zegelman

- Die neue S3-Leitlinie Multimorbidität: www.degam.de/degam-leitlinien-379.html

\section{AOK-Daten}

\section{Gesundes Altern klappt nicht}

\section{Menschen in Deutschland werden zwar immer älter, dabei aber immer öfter multimorbide, wie eine Analyse von AOK-Daten aus Niedersachsen zeigt.}

Forscher der Medizinischen Hochschule Hannover berücksichtigten für ihre Studie Personen, die zwischen 2005 und 2014 bei der AOK Niedersachsen versichert waren. Analysiert wurden alle
Diagnosen chronischer Erkrankungen bei über 60-Jährigen.

Im Jahr 2005 betrug die verbliebene Lebenserwartung für 60-jährige Frauen 24,0 und für 60-jährige Männer 19,2 Jahre. Im Jahr 2014 lag sie für 60-Jährige um 0,63 Jahre (Frauen) und 0,69 Jahre (Männer) höher. Gleichzeitig nahm der Anteil der Multimorbiden jedoch altersadjustiert drastisch zu. Erfüllten im
Jahr 2005 nur 18,6\% der Männer und $22,1 \%$ der Frauen die entsprechenden Kriterien, waren es 2014 für eine vergleichbare Altersstruktur 31,6\% der Männer und 35,0\% der Frauen. Der Anteil der Multimorbiden hatte sich innerhalb von zehn Jahren altersadjustiert folglich fast verdoppelt. $\quad$ - mut

- Tetzlaff J et al. Int J Public Health 2017;62:679-686 